\title{
Sliding Mode Congestion Controller for Data Transmission Networks with Unknown and Variable Packet Loss Rates
}

\author{
Andrzej BARTOSZEWICZ*, Pawel LATOSIŃSKI \\ Łódź University of Technology, Institute of Automatic Control, \\ Stefanowskiego 18/22, 90-942 Łódź. Poland. \\ andrzej.bartoszewicz@p.lodz.pl
}

* Corresponding author

\begin{abstract}
In this paper, the problem of data flow control in multi-source connection oriented communication networks is considered. Each source in the considered networks is characterized by its own maximum transmission rate. Furthermore, data packets sent by the sources are transferred through lossy links and some of them can be lost before arriving at a common bottleneck node. The amount of data lost during the transmission is not known, but upper bounded for each source. If at some time the bottleneck link cannot transfer all arriving data, then the excess data is stored in a buffer with limited capacity. In order to eliminate data losses caused by the buffer overflow and to ensure full utilization of the available bandwidth, in this paper a new non-switching type reaching law for discrete time sliding mode control systems is proposed and applied to design a congestion controller for the networks.
\end{abstract}

Keywords: Communication Networks, Reaching Law Approach, Discrete-Time Sliding Mode Control.

\section{Introduction}

Continuous time sliding mode controllers were introduced in Russia in late 1950s [1,2]. Soon, they have proved to be computationally efficient, easy to tune and insensitive with respect to a class of external disturbances and model uncertainties [3]. These highly desirable properties made them very attractive for the control engineering community [4-7]. The classical method of designing a sliding mode controller consists of stating the control law and proving that it ensures stability of the sliding motion. However, digital implementation of this control may result in undesirable chattering, i.e. high frequency oscillations that may cause energy loss or plant damage. In order to prevent this effect, discrete time sliding mode controllers were developed in $1980 \mathrm{~s}[8,9]$.

The introduction of discrete time quasi-sliding modes led to many further advances in the field $[10-21]$. Furuta proposed an algorithm that drives the system state to a cone-like sector defined in the state space [10]. An alternative method introduced by Gao et. al. in [11] drives the state strictly to a vicinity of the sliding hyperplane, rather than to some sector. That work presents the switching type discrete time sliding mode control, i.e. it requires the control strategy to drive the system state to the other side of the switching surface in each consecutive sampling instant. On the other hand, the equivalent control method used by Bartolini et. al. [12] drives the state to a certain neighbourhood of the sliding hyperplane without the need to cross the manifold in every sampling instant. This non-switching type discrete time sliding mode control was then studied by Bartoszewicz [13]. The width of the boundary layer in this case was further considered by $\mathrm{Su}$ et. al. [14]. Afterwards, an integral sliding mode control strategy has also been proposed in order to eliminate the reaching phase in discrete time sliding mode control [15]. An exhaustive review of the discrete time sliding mode control literature can be found in paper [16].

One of the main drawbacks of the sliding mode control methodology is the need for full information about the system state at the moment of calculating the control signal. Since this requirement often limits the applicability of such strategies, various authors have worked on that problem. Corradini and Orlando utilized the concept of time-delay control to estimate the effects of uncertainties in the switching region [17]. Bandyopadhyay and Janardhanan proposed a novel method called multirate output feedback approach [18], in which each value of the control signal is calculated based on multiple output samples. The approach was further discussed in papers [19-21]. 
In early years of sliding mode control, the design procedure included proving stability of the sliding motion by finding an appropriate Lyapunov function. However, that stage can be circumvented by utilizing an alternative method of sliding mode controller design called the reaching law approach. This approach, first proposed in [22] for continuous time systems and in [11] for discrete time ones (see [23] for further analysis), is based on stating the desired evolution of the sliding variable and applying the evolution to synthesize a feasible control law. Various authors have proposed new control methods based on the reaching law approach [24-28], which greatly improved upon the classic equivalent control or the constant-plus-proportional reaching law introduced by Gao et. al. [11]. The case of mismatched disturbance has also been considered in several works [29-31].

In recent years, the area of broadband connectivity has experienced rapid growth. Consequently, the increase of traffic intensity in data transmission networks highlighted the importance of efficient congestion control. Since the physical channel capacity does not grow as fast as the bandwidth demand, it became vital to implement new data transfer solutions. This area of research has been investigated by various authors [32-46]. An overview of earlier congestion control strategies is presented in [32]. Then, the problem of source rate synchronization present in several of the earlier algorithms has been tackled in [33]. Furthermore, an extensive new approach to flow rate controller design was proposed in [34]. In the same work, novel stochastic algorithms for data transmission networks have been proposed. Furthermore, various researchers at that time proposed strategies based on the classical PD controllers [35], PID controllers [36], adaptive methods [37] or fuzzy PID controllers [38].

An important issue that arises in congestion control of data transmission networks is the presence of long propagation delays. To address this problem, many authors have employed control schemes based on a Smith predictor. First, Mascolo introduced an algorithm that uses the difference between the queue length and its demand value as well as the number of 'in flight' packets to calculate the flow rate. This method was initially applied to a simplified case with a single virtual connection [39] and then extended to networks consisting of multiple connections [40]. Then, a method combining the Smith predictor with the PI controller was proposed [41] and shown to reduce the average bottleneck queue length. The idea of combining the Smith predictor with a proportional controller with saturation was further explored by De Cicco et. al. [42].

In recent years, several researchers have utilized sliding mode control to regulate the data flow in communication networks. Bartoszewicz and Żuk proposed to model the data transmission networks as discrete time systems with the available bandwidth acting as the disturbance [43]. In the same work, an algorithm ensuring a finite time response of the system was presented. Afterwards, various authors utilized discrete-time integral sliding mode control [44, 45], which offers good robustness at the price of relative complexity of controller design. Baburaj and Bandyopadhyay further proposed a simplified approach based on deriving a first order model of the network and applying a method based on equivalent sliding mode control [46]. An extensive review of congestion control techniques can be found in [30].

In this paper, data flow in communication networks will be regulated by means of reaching law based sliding mode control. The main contribution of such an approach is providing good robustness with respect to unpredictable bit rate variations and packet losses, while ensuring a computationally efficient controller operation. In contrast to a similar approach proposed in [30], the method introduced in this paper does not require the packet losses to be a priori known or constant. The networks considered in this work consist of several data sources and a common bottleneck link. Data sent from the sources is queued at the bottleneck link up to a certain maximum value determined by the buffer capacity. The objective of the control strategy is to ensure that the buffer capacity is never exceeded and that the available bandwidth is always fully utilized. Furthermore, the control signal should neither require the sources to send a negative amount of data, nor to exceed their maximum transmission capabilities. The upper and lower bounds of the control signal and the bottleneck queue length will be ensured by an appropriate choice of the design parameters. This will be achieved in the presence of mismatched uncertainty, 
which appears in the discrete model of the network.

\section{Communication Network Model}

In this paper, connection oriented communication networks consisting of multiple data sources, intermediate nodes and destinations are considered. Data from all $\mathrm{m}$ sources is queued at a common bottleneck link up to a certain amount determined by the buffer capacity. The bottleneck queue length is denoted by $y(k T)$, where $T$ is the sampling period. It is assumed that the queue is empty prior to the beginning of the control process, i.e. $y(k T)=0$ for $k \leq 0$. Function $d(k T)$ represents the available bandwidth. In this paper, best-effort networks with variable bit rate are considered, which means that the value of $d(k T)$ is a priori unknown, but for any $k$ it is upper bounded by a certain constant $d_{\max }$. The amount of data that actually leaves the bottleneck link at a given time is represented by $h(k T)$, where

$$
0 \leq h(k T) \leq d(k T) \leq d_{\max } .
$$

Control signal $u(k T)$ is calculated based on the current queue length and past control signals (it is assumed that $u(k T<0)=0)$. Then, the signal is distributed among all data sources according to their maximum transmission rates. If the maximum admissible transmission rate of the $j$ th source is $u_{j \max }$, then the control signal allocated to the source

$$
u_{j}(k T)=u(k T) \cdot \frac{u_{j \max }}{u_{\max }},
$$

where $u_{\max }$ is the sum of all $u_{j \max }$. Signal (2) arrives at the $j$-th source after a backward delay $T_{j \mathrm{~B}}$. Then, data is sent from the source to the bottleneck link with a forward delay $T_{j \mathrm{~F}}$. The round trip time for each source is denoted as

$$
R T T_{j}=T_{j \mathrm{~F}}+T_{j \mathrm{~B}} .
$$

Discretization period $T$ is selected to ensure that for each $j=1, \ldots, m, R T T_{j}$ is a multiple of $T$. Some of the data packets can be lost during transmission to the bottleneck link. Therefore, the amount of data from the $j$-th source that arrives at the destination at a given time $k T$ is equal to the control signal $u\left(k T-R T T_{j}\right)$ multiplied by $\alpha_{j}(k T) \in\left[\alpha_{j \min }, \alpha_{j \max }\right]$. Naturally, $\alpha_{j \min }>0$ and $\alpha_{j \max } \leq 1$ for each $j=1, \ldots, m$. It is assumed that the amount of data that can reach the bottleneck link at any moment is always strictly greater than the amount of data that can leave it, i.e.

$\sum_{j=1}^{m} \alpha_{j \min } u_{j \max }>d_{\max } \cdot$

This assumption is made to guarantee that it is always possible to maintain positive values of bottleneck queue length. It is a necessary (albeit not sufficient) condition for ensuring full utilization of the available bandwidth. The queue length at any sampling instant

$y(k T)$

$=\sum_{l=0}^{k-1} \sum_{j=1}^{m} \alpha_{j}(l T) u_{j}\left(l T-R T T_{j}\right)-\sum_{l=0}^{k-1} h(l T)$.

In order to simplify the model, all connections with equal round trip times are first grouped together and expressed as a single virtual connection. The amount of data arriving at the bottleneck from each virtual connection at the moment $k T$ is $\beta_{r}(k T) u(k T-r T)$, where

$\beta_{r}(k T)=\sum_{j: R T T_{j}=r T} \alpha_{j}(k T) \cdot \frac{u_{j \max }}{u_{\max }}$.

for $r=1, \ldots, \max \left(R T T_{j} / T\right)$. When there are no sources such that $R T T_{j} / T=r$ for a given $r$, then $\beta_{r}(k T)=0$. Denoting $n=\max \left(R T T_{j} / T\right)+1$, one can express the queue length $y$ as

$y(k T)$

$=\sum_{l=0}^{k-1} \sum_{r=1}^{n-1} \beta_{r}(l T) u(l T-r T)-\sum_{l=0}^{k-1} h(l T)$.

For each $r=1, \ldots, n-1, \quad$ coefficients $\beta_{r \text { min }}, \beta_{r \text { max }}, \bar{\beta}_{\mathrm{r}}, \delta \beta_{r}$ are defined in the following way

$\beta_{r \min }=\sum_{j: R T T_{j}=r T} \alpha_{j \min } \cdot \frac{u_{j \max }}{u_{\max }}$,

$\beta_{r \text { max }}=\sum_{j: R T T_{j}=r T} \alpha_{j \max } \cdot \frac{u_{j \max }}{u_{\max }}$,

$\bar{\beta}_{r}=\left(\beta_{r \text { max }}+\beta_{r \text { min }}\right) / 2$,

$\delta \beta_{r}=\left(\beta_{r \text { max }}+\beta_{r \text { min }}\right) / 2$.

Next, the following representation of the considered network is obtained. System dynamics are expressed in the state space as 


$$
\begin{gathered}
\boldsymbol{x}[(k+1) T]=\boldsymbol{A} \boldsymbol{x}(k T)+\tilde{\boldsymbol{A}}(k T) \boldsymbol{x}(k T) \\
+\boldsymbol{b} u(k T)+\boldsymbol{p} h(k T) \\
y(k T)=\boldsymbol{q}^{\mathrm{T}} \boldsymbol{x}(k T) .
\end{gathered}
$$

The first state variable of the $n$ dimensional state vector $\boldsymbol{x}(k T)$ is the queue length $y(k T)$. The remaining variables represent delayed control signals. In other words, for any $i=1, \ldots, n$

$$
x_{i}(k T)= \begin{cases}y(k T) & \text { for } i=1 \\ u[(k-n+i-1) T] & \text { for } \quad i>1\end{cases}
$$

The $n \times n$ dimensional state matrix

$$
\boldsymbol{A}=\left[\begin{array}{ccccc}
1 & \beta_{n-1 \max } & \beta_{n-2 \max } & \cdots & \beta_{1 \max } \\
0 & 0 & 1 & & 0 \\
\vdots & & & \ddots & \vdots \\
0 & 0 & 0 & & 1 \\
0 & 0 & 0 & \cdots & 0
\end{array}\right]
$$

and matrix representing parameter uncertainties

$$
\tilde{A}(k T)=\left[\begin{array}{cccc}
0 & \tilde{a}_{n-1}(k T) & \cdots & \tilde{a}_{1}(k T) \\
0 & 0 & & 0 \\
\vdots & & \ddots & \vdots \\
0 & 0 & \cdots & 0
\end{array}\right],
$$

where for every $i=1, \ldots, n-1$

$$
\tilde{a}_{i}(k T)=\beta_{i}(k T)-\beta_{i \max } .
$$

Moreover, $n \times 1$ dimensional vectors $\boldsymbol{b}, \boldsymbol{p}, \boldsymbol{q}$ are

$$
\boldsymbol{b}=\left[\begin{array}{c}
0 \\
\vdots \\
0 \\
1
\end{array}\right], \quad \boldsymbol{p}=\left[\begin{array}{c}
-1 \\
0 \\
\vdots \\
0
\end{array}\right], \quad \boldsymbol{q}=\left[\begin{array}{c}
1 \\
0 \\
\vdots \\
0
\end{array}\right]
$$

Finally, $h(k T) \in\left[0, d_{\max }\right]$ is an a priori unknown scalar function representing the disturbance. A diagram illustrating the considered class of networks is shown in Figure 1. In the next section, this model will be utilized to design a congestion controller for the network.

\section{Control Strategy}

The goal of the control strategy is to ensure the following properties of the system:
- Control signal $u$ must be non-negative and upper bounded due to limited data transfer rate.

- Queue length y must be upper bounded for all time instants due to the limited data buffer size capacity.

- After $\max \left(R T T_{j} / T\right)$ initial steps, queue length $y$ must be strictly positive to ensure that the available bandwidth is fully utilized.

To that end, the strategy will lead the system state $\boldsymbol{x}(k T)$ to a certain desired value $\boldsymbol{x}_{d}$. Vector $\boldsymbol{x}_{d}$ is defined as $\left[y_{d} 0 \ldots 0\right]^{\mathrm{T}}$, where $y_{d}$ is a positive constant selected by the designer. The choice of $y_{d}$ will be discussed in greater detail later in this paper. Let the sliding surface be defined as

$s(k T)$
$=\boldsymbol{c}^{\mathrm{T}} \boldsymbol{x}_{d}-\boldsymbol{c}^{\mathrm{T}} \boldsymbol{x}(k T)=c_{1} y_{d}-\boldsymbol{c}^{\mathrm{T}} \boldsymbol{x}(k T)=0$.

Vector $c$ in relation (15) is selected to ensure a finite time response of the discrete system [43], which means it must satisfy

$$
\operatorname{det}\left\{z \boldsymbol{I}_{n}-\left[\boldsymbol{I}_{n}-\boldsymbol{b}\left(\boldsymbol{c}^{\mathrm{T}} \boldsymbol{b}\right)^{-1} \boldsymbol{c}^{\mathrm{T}}\right] \boldsymbol{A}\right\}=z^{n}
$$

where $\boldsymbol{c}^{\mathrm{T}} \boldsymbol{b} \neq 0$. Substituting parameters (11) and (14) into (16), we obtain elements of vector $c$

$c_{i}= \begin{cases}1 & \text { for } \quad i=1 \\ \sum_{r=1}^{i-1} \beta_{n-r \max } & \text { for } \quad i=2, \ldots, n .\end{cases}$

In this paper, a new sliding mode control strategy for communication networks (9) will be obtained by means of reaching law approach. In other words, the desired evolution of the sliding variable $s(k T)$ will first be defined in the form of a reaching law, and then the control signal will be calculated from the law.

\subsection{Proposed reaching law based strategy}

We propose the following original reaching law for the considered network

$$
\begin{aligned}
& s[(k+1) T]=s(k T)-c^{\mathrm{T}} \tilde{\boldsymbol{A}}(k T) \boldsymbol{x}(k T) \\
& +h(k T)-F-s_{0} f[s(k T)] \operatorname{sgn}[s(k T)],
\end{aligned}
$$




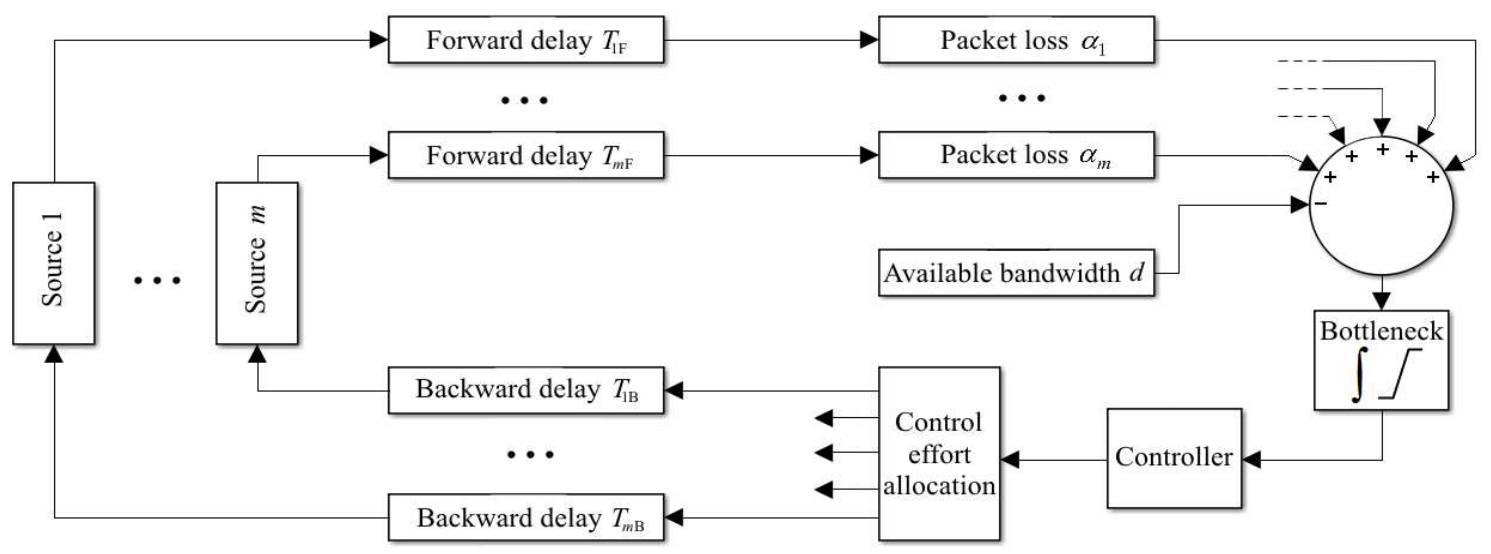

Figure 1: Network Model

where $s_{0}$ is the design parameter, $f$ is a non-negative upper bounded by 1 function expressed as

$$
f[s(k T)]=1-\exp \left[-\frac{s(k T)^{2}}{s_{0}{ }^{2}}\right]
$$

and $F$ is a positive constant

$$
F=\frac{d_{\max }}{2}+u_{\max } \sum_{r=1}^{n-1} \delta \beta_{r} .
$$

It is easy to notice that if $|s(k T)|$ is relatively large, the absolute value of the sliding variable will decrease by approximately $s_{0}$ in the next step. Thus, the proposed reaching law ensures a fast convergence of the sliding variable to the vicinity of 0 . On the other hand, for small values of $|s(k T)|$, the term $s_{0} f[s(k T)]$ decreases, which in turn reduces the sliding variable rate of change when the variable is in the vicinity of 0 . In order to derive the control signal from the proposed reaching law, $s[(k+1) T]$ is first substituted from (15) into (18)

$$
\begin{aligned}
& y_{d}-c^{\mathrm{T}} \boldsymbol{x}[(k+1) T]=s(k T)-\boldsymbol{c}^{\mathrm{T}} \tilde{\boldsymbol{A}}(k T) \boldsymbol{x}(k T) \\
& +h(k T)-F-s_{0} f[s(k T)] \operatorname{sgn}[s(k T)],
\end{aligned}
$$

Further substituting $\boldsymbol{x}[(k+1) T]$ from (9) into (21) and solving for $u(k T)$, one obtains

$$
\begin{aligned}
& u(k T)=-\left(\boldsymbol{c}^{\mathrm{T}} \boldsymbol{b}\right)^{-1}\left\{s(k T)-y_{d}-F\right. \\
& \left.-s_{0} f[s(k T)] \operatorname{sgn}[s(k T)]+\boldsymbol{c}^{\mathrm{T}} \boldsymbol{A} \boldsymbol{x}(k T)\right\} .
\end{aligned}
$$

The obtained control law can be simplified taking the known system parameters into account. To that end, the following fact will be brought up in the form of a lemma.
Lemma 1: If matrix $\boldsymbol{A}$ and vector $\boldsymbol{c}$ are defined by (11) and (17) respectively, then $\boldsymbol{c}^{\mathrm{T}} \boldsymbol{A x}=\boldsymbol{c}^{\mathrm{T}} \boldsymbol{x}$ for each state $\boldsymbol{x}$.

Proof: For the sake of clarity, proof is given in Appendix 1.

Taking Lemma 1 and relations (14) and (17) into account, control law (22) can be rewritten in the following way

$u(k T)$

$=\left(\sum_{r=1}^{n-1} \beta_{r \max }\right)^{-1}\left\{s_{0} f[s(k T)] \operatorname{sgn}[s(k T)]+F\right\}$.

Since total data transmission capabilities of all $m$ sources are limited by $u_{\max }$, parameter $s_{0}$ in relation (23) should be selected in a way that imposes a global upper bound $u_{\max }$ on the control signal. The following theorem provides the parameter which satisfies this criterion.

\section{Theorem 1: If}

$s_{0}=u_{\max }\left(\sum_{r=1}^{n-1} \beta_{r \max }\right)^{-1}-F$,

then for any time $k T$ the control signal $u(k T)$ is not greater than $u_{\max }$. Furthermore, $s_{0}$ selected in such a way is always strictly greater than $F$.

Proof: First it will be shown that the control signal $u(k T)$ is upper bounded by $u_{\max }$. Since for any $s(k T)$ function $f$ is upper bounded by 1 , it follows from (23) that

$$
u(k T) \leq\left(\sum_{r=1}^{n-1} \beta_{r \max }\right)^{-1}\left(s_{0}+F\right),
$$

Substituting $s_{0}$ from (24) into (25), one obtains $u(k T) \leq u_{\max }$, which proves the first part of the 
theorem. Next, it will be shown that the selected $s_{0}$ is strictly greater than $F$. Indeed

$$
\begin{aligned}
s_{0} & =u_{\max } \sum_{r=1}^{n-1}\left(\beta_{r \min }+\beta_{r \max }-\beta_{r \min }\right)-F \\
& =u_{\max } \sum_{r=1}^{n-1} \beta_{r \min }+u_{\max } \sum_{r=1}^{n-1} 2 \delta \beta_{r}-F \\
& =\sum_{j=1}^{m} \alpha_{j \min } u_{j \max }+2 u_{\max } \sum_{r=1}^{n-1} \delta \beta_{r}-F .
\end{aligned}
$$

Furthermore, taking relation (4) into account, one obtains

$$
\begin{aligned}
s_{0} & >d_{\max }+2 u_{\max } \sum_{r=1}^{n-1} \delta \beta_{r}-F \\
& =2 F-F=F .
\end{aligned}
$$

\subsection{Properties of the strategy}

It will be shown that reaching law (18) ensures that once the system state enters a certain band around the sliding plane, it will remain inside the band for all future sampling instants. Furthermore, when the state is out of the band, it will approach the band at least asymptotically. In order to prove those properties, a helpful fact in the form of a lemma is first demonstrated.

Lemma 2: If $s_{1} \leq s_{2}\left(s_{1}, s_{2} \in \mathrm{R}\right)$, then for any $s_{0}>0$ the following inequality is satisfied

$$
\begin{aligned}
& s_{1}-s_{0} f\left(s_{1}\right) \operatorname{sgn}\left(s_{1}\right) \\
& \leq s_{2}-s_{0} f\left(s_{2}\right) \operatorname{sgn}\left(s_{2}\right)
\end{aligned}
$$

Proof: For the sake of clarity, proof is given in Appendix 2.

It will first be shown that the sliding variable is always lower bounded. To that end, the following theorem will be proven.

Theorem 2: If $s_{0}$ is selected according to (24), then for any $k$

$$
s(k T) \geq-q=-s_{0} \sqrt{\ln \left(\frac{s_{0}}{s_{0}-F}\right)} .
$$

Proof: The proof is based on the principle of strong mathematical induction. If $s(0) \geq-q$ and the implication

$$
\forall_{l=0, \ldots, k} s(l T) \geq-q \Rightarrow s[(k+1) T] \geq-q
$$

holds for any $k \geq 0$, then one can conclude from strong mathematical induction that $s(k T) \geq-q$ for all $k$. Relation (14) gives $s(k T)=y_{d}-$ $c^{\mathrm{T}} \boldsymbol{x}(k T)$. Since for the considered class of systems $\boldsymbol{x}(0)$ is always equal to $\mathbf{0}$, one gets $s(0)=y_{d}>0>-q$. It is therefore sufficient to show that (30) is true for each $k$. Let $k$ be an arbitrarily selected natural number and let $s(l T) \geq-q$ for $l=0, \ldots, k$. Since $s(k T) \geq-q$, Lemma 2 implies

$$
\begin{aligned}
& -q+s_{0} f(-q) \\
& \leq s(k T)-s_{0} f[s(k T)] \operatorname{sgn}[s(k T)] .
\end{aligned}
$$

Consequently, relation (18) gives

$$
\begin{aligned}
s[(k+1) T] \geq & -q-c^{\mathrm{T}} \tilde{\boldsymbol{A}}(k T) \boldsymbol{x}(k T) \\
& +h(k T)-F+s_{0} f(-q) .
\end{aligned}
$$

From (19) it can be seen that

$s_{0} f(-q)=s_{0}\left[1-\exp \left(-\frac{q^{2}}{s_{0}^{2}}\right)\right]=F$.

Substitution of (33) into (32) yields

$$
\begin{aligned}
s[(k+1) T] \geq & -q-c^{\mathrm{T}} \tilde{\boldsymbol{A}}(k T) \boldsymbol{x}(k T) \\
& +h(k T) .
\end{aligned}
$$

As stated in (10), state variables $x_{2}, \ldots, x_{n}$ represent delayed control signals. Therefore, relations (12) and (17) give

$\boldsymbol{c}^{\mathrm{T}} \tilde{\boldsymbol{A}}(k T) \boldsymbol{x}(k T)$

$=\sum_{r=1}^{n-1}\left[\beta_{r}(k T)-\beta_{r \max }\right] \cdot u[(k-r) T]$,

where $\beta_{r}(k T)-\beta_{r \max } \leq 0$ for each $r$. Since $s(l T) \geq-q$ for $l=0, \ldots, k$ and function $f[s(k T)] \operatorname{sgn}[s(k T)]$ is strictly increasing with respect to $s(k T)$, relation (23) implies

$$
\begin{aligned}
u(l T) & \geq\left(\sum_{r=1}^{n-1} \beta_{r \max }\right)^{-1}\left[-s_{0} f(-q)+F\right] \\
& =\left(\sum_{r=1}^{n-1} \beta_{r \max }\right)^{-1}(-F+F)=0
\end{aligned}
$$

for $l=0, \ldots, k$. Furthermore, it has already been stated that $u(k T<0)=0$. Consequently, relation (35) gives 


$$
\boldsymbol{c}^{\mathrm{T}} \tilde{\boldsymbol{A}}(k T) \boldsymbol{x}(k T) \leq 0 .
$$

Moreover, for any $k$ relation (1) implies $h(k T) \geq 0$. Taking that fact and inequality (37) into account, (34) becomes

$$
s[(k+1) T] \geq-q-0+0=-q .
$$

Therefore, implication (30) holds for the selected $k$. Since $k$ was chosen arbitrarily, one can conclude that (30) is always true. Moreover, since $s(0)>-q$, the principle of strong mathematical induction gives $s(k T) \geq-q$ for any $k$.

Remark 1: Theorem 2 has shown that $s(k T)$ $\geq-q$ for all $k \geq 0$. Therefore, relation (36) will remain true for any $k$ and consequently, the obtained lower bound of the control signal becomes global. That fact, together with Theorem 1, imply that the control signal generated by the proposed strategy will always be non-negative and upper bounded by $u_{\max }$. Therefore, the strategy will never require the sources to send a negative amount of data or to exceed their transmission capabilities.

It has been demonstrated that the sliding variable is always lower bounded. It will now be shown that if the variable enters a certain band around the switching plane, it will remain inside the band for all subsequent sampling instants.

Theorem 3: If $s_{0}$ is chosen as stated in (24) and

$$
s(k T) \leq q=s_{0} \sqrt{\ln \left(\frac{s_{0}}{s_{0}-F}\right)}
$$

for a certain $k$, then $s[(k+1) T] \leq q$.

Proof: Let $s(k T)$ be not greater than $q$. Lemma 2 gives

$$
\begin{aligned}
& s(k T)-s_{0} f[s(k T)] \operatorname{sgn}[s(k T)] \\
& \leq q-s_{0} f(q) .
\end{aligned}
$$

Since function $f$ is even, (33) implies $s_{0} f(q)=s_{0} f(-q)=F$. Furthermore, Remark 1 and Theorem 1 state that the control signal is always non-negative and upper bounded by $u_{\max }$. Consequently, the expression on the right hand side of (35) will always be non-positive. Thus, relations (8) and (35) give

$$
\begin{aligned}
\boldsymbol{c}^{\mathrm{T}} \tilde{\boldsymbol{A}}(k T) \boldsymbol{x}(k T)+u_{\max } \sum_{r=1}^{n-1} \delta \beta_{r} \\
=\sum_{r=1}^{n-1}\left[\beta_{r}(k T)-\beta_{r \max }\right] \cdot u[(k-r) T] \\
\quad+u_{\max } \sum_{r=1}^{n-1} \delta \beta_{r} \\
\geq u_{\max } \sum_{r=1}^{n-1}\left[\beta_{r}(k T)-\beta_{r \max }+\delta \beta_{r}\right] \\
=u_{\max } \sum_{r=1}^{n-1}\left[\beta_{r}(k T)-\bar{\beta}_{r}\right] \\
\geq-u_{\max } \sum_{r=1}^{n-1} \delta \beta_{r} .
\end{aligned}
$$

Moreover, relation (1) implies

$\left|d(k T)-d_{\max } / 2\right| \leq d_{\max } / 2$.

Taking inequalities (40), (41) and (42) into account, substitution of (20) into (18) yields

$$
\begin{aligned}
& s[(k+1) T] \\
\leq & q-c^{\mathrm{T}} \tilde{\boldsymbol{A}}(k T) \boldsymbol{x}(k T)+h(k T)-F-s_{0} f(q) \\
= & q-\boldsymbol{c}^{\mathrm{T}} \tilde{\boldsymbol{A}}(k T) \boldsymbol{x}(k T)-u_{\max } \sum_{r=1}^{n-1} \delta \beta_{r} \\
& +h(k T)-d_{\max } / 2-F \\
\leq & q+u_{\max } \sum_{r=1}^{n-1} \delta \beta_{r}+d_{\max } / 2-F \\
& q+F-F=q .
\end{aligned}
$$

Taking Theorem 2 and Theorem 3 into consideration, one concludes that if the system state enters a quasi-sliding mode band

$\left\{\boldsymbol{x}:\left|y_{d}-\boldsymbol{c}^{\mathrm{T}} \boldsymbol{x}\right| \leq q=s_{0} \sqrt{\ln \left(\frac{s_{0}}{s_{0}-F}\right)}\right\}$

then it will remain inside the band in all subsequent sampling instants. Furthermore, Theorem 2 states that the sliding variable can never assume values smaller than $-q$. Consequently, if the system state is out of the band, then the sliding variable must be greater than $q$. The following theorem will demonstrate that if $s(k T)>q$, then the state will approach the band (44) at least asymptotically.

Theorem 4: If $\boldsymbol{x}(k T)$ is such a state that $s(k T)>q$, then the system state will reduce its distance from the band (44) in every subsequent step and either enter the band in finite time or approach it asymptotically.

Proof: Let $s(k T)$ be greater than $q$. Then, relation (19) implies $f[s(k T)]>f(q)$. Furthermore, from (41) and (42) one obtains 
$s[(k+1) T]$

$<s(k T)-s_{0} f(q)+d_{\max } / 2+u_{\max } \sum_{r=1}^{n-1} \delta \beta_{r}$

$\leq s(k T)-F+F=s(k T)$.

Therefore, when $s(k T)$ is greater than $q$, the system state will approach the band (44) in the next step. Consequently, the state will either enter the band in finite time or converge asymptotically to a certain positive value. Theorem 3 states that if the state enters the band, it will remain inside it for all future steps. On the other hand, if $s(k T)$ asymptotically approaches a certain value $q_{+}>0$, then

$$
\begin{aligned}
& \lim _{k \rightarrow \infty} s[(k+1) T] \\
& \leq \lim _{k \rightarrow \infty}\left\{s(k T)-s_{0} f[s(k T)]+F\right\} \\
& =q_{+}-s_{0} f\left(q_{+}\right)+F .
\end{aligned}
$$

As $s[(k+1) T]$ also converges to $q_{+},(46)$ gives

$$
q_{+} \leq q_{+}-s_{0} f\left(q_{+}\right)+F .
$$

Solving (47) for $q_{+}$, one obtains $q_{+} \leq q$, which means that if $s(k T)>q$, the system state is either confined to the band (44) in finite time or, in the worst possible case, asymptotically approaches the band.

As stated at the beginning of this section, it is required that after a certain finite amount of steps, the queue length $y(k T)$ is strictly greater than 0 to guarantee that the available bandwidth is fully utilized in every step. This property is ensured by the appropriate choice of target queue length $y_{d}$ as stated in the following theorem.

Theorem 5: If $k>\max \left(R T T_{j} / T\right)$ and

$$
y_{d}>u_{\max } \sum_{i=2}^{n} c_{i}+q,
$$

then $y(k T)$ is always strictly greater than 0 .

Proof: Let $k$ be any natural number greater than $\max \left(R T T_{j} / T\right)$. It will be shown that $y(k T)>0$. Two cases will be considered: for $s(k T)$ outside and inside the band (44).

Case 1: First let $s(k T)>q$. Theorem 4 states that $s(l T)>\mathrm{s}(k T)$ for each $l=0, \ldots, k-1$. Furthermore, since $f[s(k T)] \operatorname{sgn}[s(k T)]$ is increasing with respect to $s(k T)$, one obtains $u(l T)$

$=\left(\sum_{r=1}^{n-1} \beta_{r \max }\right)^{-1}\left\{s_{0} f[s(l T)] \operatorname{sgn}[s(l T)]+F\right\}$

$>\left(\sum_{r=1}^{n-1} \beta_{r \max }\right)^{-1}\left[s_{0} f(q)+F\right]=2 F\left(\sum_{r=1}^{n-1} \beta_{r \max }\right)^{-1}$

for $l=0, \ldots, k-1$. Moreover, relation (7) implies

$$
\begin{aligned}
y(k T)= & y[(k-1) T]-h[(k-1) T] \\
& +\sum_{r=1}^{n-1} \beta_{r}(k T) u(k T-r T) .
\end{aligned}
$$

Since $k>\max \left(R T T_{j} / T\right)=n-1$, all elements $u(k T-r T)$ in relation (50) are lower bounded as stated in (49). Furthermore, it is known from (1) that $h[(k-1) T]$ is upper bounded by $d_{\max }$. Finally, the queue length $y[(k-1) T]$ is always non-negative. Consequently, relation (50) implies

$$
\begin{aligned}
y(k T) & >2 F\left[\sum_{r=1}^{n-1} \beta_{r}(k T)\right]\left(\sum_{r=1}^{n-1} \beta_{r \max }\right)^{-1}-d_{\max } \\
& \geq 2 F\left(\sum_{r=1}^{n-1} \beta_{r \text { min }}\right)\left(\sum_{r=1}^{n-1} \beta_{r \text { max }}\right)^{-1}-d_{\text {max }} .
\end{aligned}
$$

Substituting $F$ from (20) into (51) and further substituting $\beta_{r \min }$ from (8) into (51), one obtains

$$
\begin{aligned}
& y(k T)>d_{\max }\left(\sum_{r=1}^{n-1} \beta_{r \min }\right)\left(\sum_{r=1}^{n-1} \beta_{r \max }\right)^{-1}-d_{\max } \\
& +2\left(\sum_{j=1}^{m} \alpha_{j \min } u_{j \max }\right)\left(\sum_{r=1}^{n-1} \delta \beta_{r}\right)\left(\sum_{r=1}^{n-1} \beta_{r \max }\right)^{-1} .
\end{aligned}
$$

Relation (52), together with (4), give

$$
\begin{aligned}
& y(k T)>d_{\max }\left(\sum_{r=1}^{n-1} \beta_{r \min }\right)\left(\sum_{r=1}^{n-1} \beta_{r \text { max }}\right)^{-1}-d_{\text {max }} \\
& +2 d_{\max }\left(\sum_{r=1}^{n-1} \delta \beta_{r}\right)\left(\sum_{r=1}^{n-1} \beta_{r \text { max }}\right)^{-1} \\
& =d_{\max }\left[\left(\sum_{r=1}^{n-1} \beta_{r \text { max }}\right)^{-1}\left(\sum_{r=1}^{n-1} 2 \delta \beta_{r}+\beta_{r \text { min }}\right)-1\right]
\end{aligned}
$$

$=0$.

Thus, when $s(k T)>q$ for $k>\max \left(R T T_{j} / T\right)$, the queue length is always strictly positive.

Case 2: Now let $s(k T) \leq q$. Relation (15) gives 
$y_{d}-y(k T)-\sum_{i=2}^{n} c_{i} u[(k-n+i-1) T] \leq q$.

Theorem 1 states that for the selected $s_{0}$, control signal is always upper bounded by $u_{\max }$. Therefore, relation (54) yields

$$
\begin{aligned}
y(k T) & \geq y_{d}-\sum_{i=2}^{n} c_{i} u[(k-n+i-1) T]-q \\
& \geq y_{d}-u_{\max } \sum_{i=2}^{n} c_{i}-q .
\end{aligned}
$$

When inequality (48) is satisfied, the right hand side of relation (55) is always strictly positive. In conclusion, if $y_{d}$ is selected according to (48), then bottleneck queue length $y(k T)$ is strictly positive for all $k>\max \left(R T T_{j} / T\right)$.

Finally, the problem of limited data buffer size will be considered. Since one of the objectives is to prevent the loss of data at the bottleneck link, the strategy must ensure that the queue length is upper bounded for all time instants. That property will be demonstrated in the following theorem.

Theorem 6: For any $k \geq 0$, the following inequality is satisfied

$$
y(k T) \leq y_{d}+q,
$$

Proof: Substitution of $s(k T)$ from (15) into (29) yields

$$
y(k T)-y_{d}+\sum_{i=2}^{n} c_{i} u[(k-n+i-1) T] \leq q .
$$

Remark 1 states that $u(k T)$ is always nonnegative. Therefore

$$
y(k T)-y_{d} \leq q .
$$

Thus, inequality (55) is always satisfied.

\section{Simulation Example}

The effectiveness of the proposed method will be demonstrated by means of a simulation example. The strategy will be applied to a network consisting of 4 data sources.

Parameters of the sources are shown in Table I. The sampling period $T=0.1 \mathrm{~ms}$. Total amount of data that can be sent by all sources during the selected sampling period is $u_{\max }=10 \mathrm{Mb}$. The available data buffer capacity, i.e. the maximum amount of data queued at the bottleneck equals $40 \mathrm{Mb}$. For each $j$, the fraction of data that actually arrives at the bottleneck link is

$\alpha_{j}(k T)$

$=\frac{\alpha_{j \max }+\alpha_{j \min }}{2}-\frac{\alpha_{j \max }-\alpha_{j \min }}{2} \cdot(-1)^{\lfloor k T / 50\rfloor}$.

The maximum amount of data that can leave the bottleneck link buffer at any given time $d_{\max }=6 \mathrm{Mb}$ (which satisfies (4)). The available bandwidth is described by

$d(k T)$

$=3+1.5 \cdot(-1)^{\lfloor k T / 50\rfloor}-1.5 \cdot(-1)^{\lfloor k T / 25\rfloor}$.

Naturally, the actual disturbance $h(k T)$ will be equal to its upper bound $d(k T)$ unless the amount of data at the bottleneck link is smaller than $d(k T)$. However, according to Theorem 5, this can only occur in the first 3 time instants. Sources with the same round trip times are grouped together according to (6). Then, coefficients $\beta_{r}$ are calculated according to (8) for $r=1,2,3$. The results are shown in Table 2.

Table 1. Parameters of the Sources

\begin{tabular}{|c|c|c|c|c|}
\hline Parameter & $\begin{array}{c}\text { Source } \\
1\end{array}$ & $\begin{array}{c}\text { Source } \\
2\end{array}$ & $\begin{array}{c}\text { Source } \\
3\end{array}$ & $\begin{array}{c}\text { Source } \\
4\end{array}$ \\
\hline$u_{j \max }$ & 3 & 2 & 3 & 2 \\
\hline$T_{j \mathrm{~F}}$ & 0.03 & 0.05 & 0.07 & 0.08 \\
\hline$T_{j \mathrm{~B}}$ & 0.07 & 0.15 & 0.13 & 0.22 \\
\hline$R T T_{j}$ & 0.1 & 0.2 & 0.2 & 0.3 \\
\hline$\alpha_{j \min }$ & 0.6 & 0.6 & 0.7 & 0.5 \\
\hline$\alpha_{j \max }$ & 1 & 0.8 & 0.9 & 1 \\
\hline
\end{tabular}

Table 2. Virtual Connection Coefficients

\begin{tabular}{|c|c|c|c|}
\hline Parameter & VC1 & VC2 & VC3 \\
\hline$\beta_{r \max }$ & 0.3 & 0.43 & 0.2 \\
\hline$\beta_{r \min }$ & 0.18 & 0.33 & 0.1 \\
\hline$\beta_{r}$ & 0.24 & 0.38 & 0.15 \\
\hline$\delta \beta_{r}$ & 0.06 & 0.05 & 0.05 \\
\hline
\end{tabular}

Matrix $\boldsymbol{A}(\operatorname{dim} \boldsymbol{A}=4 \times 4)$ is defined as shown in (11) and vector $\boldsymbol{c}$ is selected according to (16). Coefficient $F$, calculated according to (19), equals $4.6 \mathrm{Mb}$. Theorem 1 determines $s_{0}=4.7 \mathrm{Mb}>F$. Consequently from (29) we get $q=9.2222 \mathrm{Mb}$. The choice of the demand queue length $y_{d}$ is restricted by (48), which means that $y_{d}>30.1222 \mathrm{Mb}$. On the other hand, Theorem 6 
states that the queue length is upper bounded by $y_{d}+q$. Since in this example that sum must not exceed $40 \mathrm{Mb}$, the demand queue length is set to $y_{d}=40 \mathrm{Mb}-q=30.7778 \mathrm{Mb}$. Finally, control law (23) determines the amount of data sent in each sampling instant. Figure 2 illustrates the evolution of the sliding variable, Figure 3 shows the bottleneck queue length and Figure 4 presents the control signal.

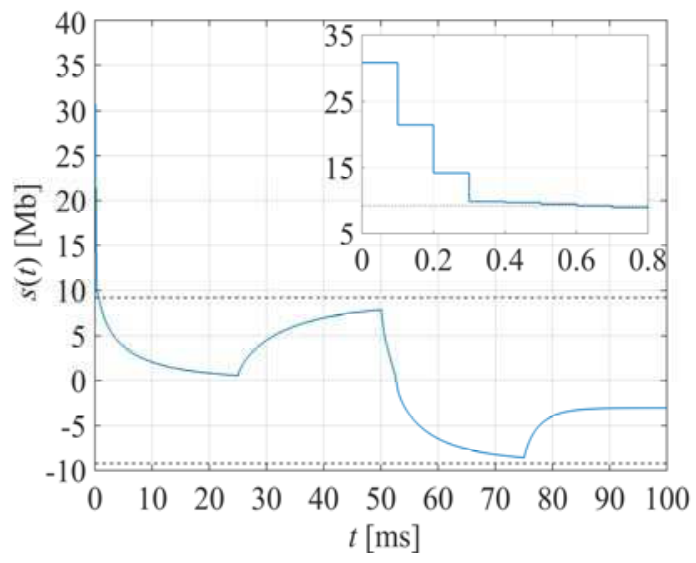

Figure 2. Sliding Variable

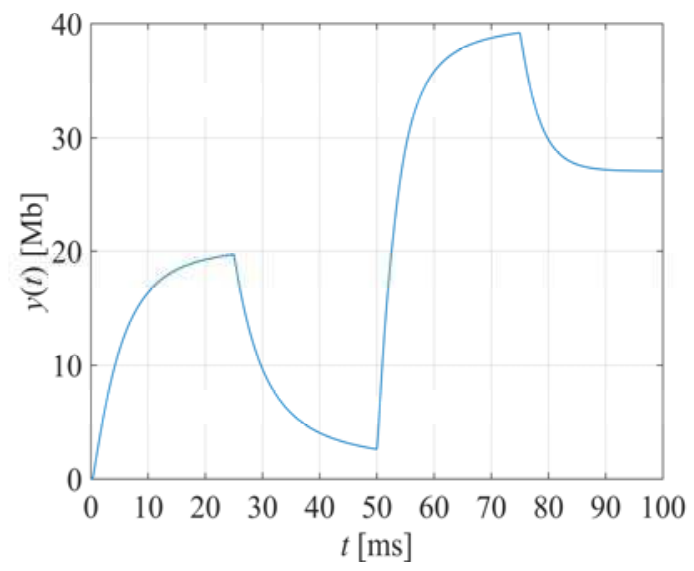

Figure 3. Bottleneck Queue Length

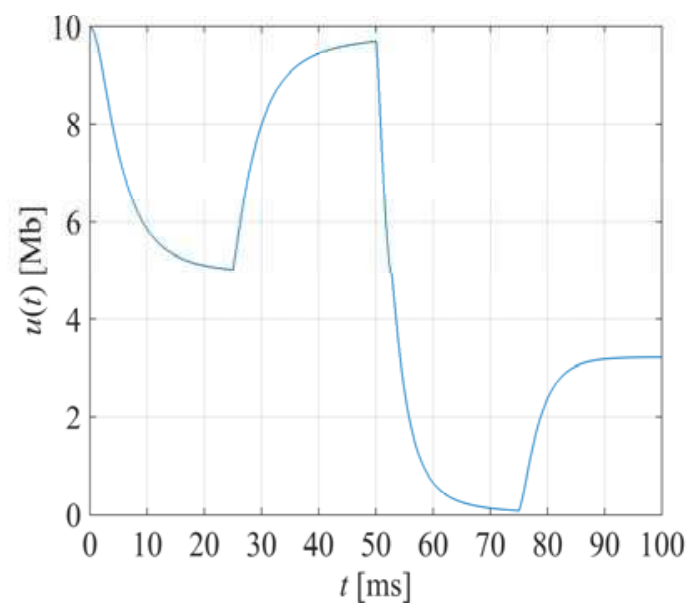

Figure 4. Control Signal
It can be seen from Figure 2 that the sliding variable quickly enters the band (44) presented with black dashed lines and remains inside it in all subsequent sampling instants. Figure 3 shows that bottleneck queue length is always positive and upper bounded by $40 \mathrm{Mb}$, which means that the available bandwidth is fully utilized and no data is lost at the bottleneck link. Finally, it can be seen from Figure 4 that the control signal never exceeds $10 \mathrm{Mb}$ and is always non-negative.

\section{Conclusions}

In this paper, a new reaching law for connection oriented communication networks has been proposed. Then, the reaching law has been utilized to obtain a sliding mode control strategy for the considered networks. It has been demonstrated that the proposed strategy ensures full utilization of the available bandwidth. Furthermore, the strategy guarantees that data transmission capabilities of the sources are not exceeded and that no data is lost at the bottleneck link.

\section{Appendix 1}

Proof of Lemma 1: It will be shown that for the matrix $\boldsymbol{A}$ defined by (11) and vector $\boldsymbol{c}$ defined by (17), equality $\boldsymbol{c}^{\mathrm{T}} \boldsymbol{A} \boldsymbol{x}(k T)=\boldsymbol{c}^{\mathrm{T}} \boldsymbol{x}(k T)$ holds for any $\boldsymbol{x}(k T)$. Left hand side of the equality is first expressed as

$$
\begin{aligned}
& \boldsymbol{c}^{\mathrm{T}} \boldsymbol{A} \boldsymbol{x}(k T) \\
& =\boldsymbol{c}^{\mathrm{T}}\left(\boldsymbol{A}-\boldsymbol{I}_{n}\right) \boldsymbol{x}(k T)+\boldsymbol{c}^{\mathrm{T}} \boldsymbol{x}(k T) .
\end{aligned}
$$

Relation (11) implies

$$
\begin{aligned}
& \boldsymbol{c}^{\mathrm{T}}\left(\boldsymbol{A}-\boldsymbol{I}_{n}\right) \\
& =\left[\begin{array}{llll}
0 & c_{1} \beta_{n-1 \max }-c_{2} & \ldots & c_{1} \beta_{1 \max }+c_{n-1}-c_{n}
\end{array}\right] .
\end{aligned}
$$

Substituting values of $c_{i}$ from (17) into (62), one obtains

$$
\boldsymbol{c}^{\mathrm{T}}\left(\boldsymbol{A}-\boldsymbol{I}_{n}\right)=\left[\begin{array}{lllll}
0 & 0 & 0 & \ldots & 0
\end{array}\right] .
$$

Therefore, the right hand side of (61) becomes $\boldsymbol{c}^{T} \boldsymbol{x}(k T)$.

\section{Appendix 2}

Proof or Lemma 2: It will be shown that the function $g(z)=z-s_{0} f(z) \operatorname{sgn}(z)$ is always 
increasing with respect to $z$. It is sufficient to show that

$$
\frac{d}{d z} g(z)=1-2 \frac{z}{s_{0}} \exp \left(-\frac{z^{2}}{s_{0}{ }^{2}}\right) \operatorname{sgn}(z)
$$

is always strictly greater than 0 . To that end, it will be demonstrated that the global minimum of (64) is positive. The only points where the minima can occur are the solutions of

$$
\begin{aligned}
& \frac{d^{2}}{d z^{2}} g(z) \\
& =\frac{2}{s_{0}{ }^{3}}\left(2 z^{2}-{s_{0}}^{2}\right) \exp \left(-\frac{z^{2}}{s_{0}^{2}}\right) \operatorname{sgn}(z)=0,
\end{aligned}
$$

which are $z= \pm s_{0} / \sqrt{2}$. Indeed, since

$$
\frac{d^{3}}{d z^{3}} g\left( \pm s_{0} / \sqrt{2}\right)=\frac{4 \sqrt{2}}{e^{2} s_{0}{ }^{2}}>0,
$$

global minima exist in both of those points. Furthermore,

$$
\frac{d}{d z} g\left( \pm s_{0} / \sqrt{2}\right) \approx 0.1422>0
$$

and it can be concluded that (63) is always positive. Consequently, function $g(z)$ is always increasing with respect to $z$.

\section{REFERENCES}

1. EMELYANOV, S. V., Variable Structure Control Systems, Nauka, Moscow, 1967.

2. UTKIN, V., Variable Structure Systems with Sliding Modes, IEEE Transactions on Automatic Control, vol. 22, no. 2, 1977, pp. 212-222.

3. DRAŽENOVIĆ, B., The Invariance Conditions in Variable Structure Systems, Automatica, vol. 5, no. 3, 1969, pp. 287-295.

4. EDWARDS, C., S., SPURGEON, Sliding Mode Control: Theory and Applications, Taylor\&Francis, London, 1998.

5. DECARLO, R. A., S., ŻAK, G., MATTHEWS, Variable Structure Control of Nonlinear Multivariable Systems: a Tutorial, Proceedings of the IEEE, vol. 76, no. 3, 1988, pp. 212-232.
6. SABANOVIC, A., Variable Structure Systems with Sliding Modes in Motion Control - A Survey, IEEE Transactions on Industrial Informatics, vol. 7, no. 2, 2011, pp. 212-223.

7. SHTESSEL, Y., C., EDWARDS, L., FRIDMAN, A., LEVANT, Sliding Mode Control and Observation, BirkhauserSpringer, New York, Heidelberg, Dordrecht, London, 2014.

8. MILOSAVLJEVIĆ, Ć., General Conditions for the Existence of a Quasisliding Mode on the Switching Hyperplane in Discrete Variable Structure Systems, Automation and Remote Control, vol. 46, no. 3, 1985, pp. 307-314.

9. UTKIN, V., S. V., DRAKUNOV, On Discrete-time Sliding Mode Control, IFAC Conference on Nonlinear Control, 1989, pp. 484-489.

10. FURUTA, K., Sliding Mode Control of a Discrete System, Systems \& Control Letters, vol. 14, no. 2, 1990, pp. 145-152.

11. GAO, W., Y., WANG, A., HOMAIFA, Discrete-time Variable Structure Control Systems, IEEE Transactions on Industrial Electronics, vol. 42, no. 2, 1995, pp. 117-122.

12. BARTOLINI, G., A., FERRARA, V., UTKIN, Adaptive Sliding Mode Control in Discrete-time Systems, Automatica, vol. 31, no. 5, 1995 pp. 769-773.

13. BARTOSZEWICZ, A., Discrete-time Quasi-sliding Mode Control Strategies, IEEE Transactions on Industrial Electronics, vol. 45, no. 4, 1998, pp. 633-637.

14. SU, W., V., DRAKUNOV, U., OZGUNER, An $O\left(T^{2}\right)$ Boundary Layer in Sliding Mode for Sampled-data Systems, IEEE Transactions on Automatic Control, vol. 45, no. 3,2000 , pp. 482-485.

15. ABIDI, K., J., XU, X., YU, On the Discrete-time Integral Sliding-mode Control, IEEE Transactions on Automatic Control, vol. 52, no. 4, 2007, pp. 709-715.

16. YU, X., B., WANG, X., LI, Computercontrolled Variable Structure Systems: the State-of-the-art, IEEE Transactions on Industrial Informatics, vol. 8, no. 2, 2011, pp. 197-205. 
17. CORRADINI, M. L., G., ORLANDO, Variable Structure Control of Discretized Continuous-Time Systems, IEEE Transactions on Automatic Control, vol. 43, no. 9, 1998, pp. 1329-1334.

18. BANDYOPADHYAY, B., S., JANARDHANAN, Discrete-Time Sliding Mode Control. A Multirate Output Feedback Approach, Springer-Verlag, Berlin, 2006.

19. JANARDHANAN, S., B., BANDYOPADHYAY, Output Feedback Sliding-mode Control for Uncertain Systems Using fast Output Sampling Technique, IEEE Transactions on Industrial Electronics, vol. 53, no. 5, 2006, pp. 1677-1682.

20. JANARDHANAN, S., V., KARIWALA, Multirate-output-feedback-based LQoptimal Discrete-time Sliding Mode Control, IEEE Transactions on Automatic Control, vol. 53, no. 1, 2008, pp. 367-373.

21. MEHTA, A. J., B., BANDYOPADHYAY, Frequency-shaped Sliding Mode Control Using Output Sampled Measurements, IEEE Transactions on Industrial Electronics, vol. 56, no. 1, 2008, pp. 28-35.

22. GAO, W., J., HUNG, Variable Structure Control of Nonlinear Systems: A New Approach, IEEE Transactions on Industrial Electronics, vol. 40, no. 1, 1993, pp. 45-55.

23. BARTOSZEWICZ, A., Remarks on 'Discrete-time Variable Structure Control Systems', IEEE Transactions on Industrial Electronics, vol. 43, no. 1, 1996, pp. 235-238.

24. GOLO, G., Ć., MILOSAVLJEVIĆ, Robust Discrete-time Chattering Free Sliding Mode Control, Systems \& Control Letters, vol. 41, no. 1, 2000, pp. 19-28.

25. MILOSAVLJEVIĆ, Ć., B., PERUNIČIĆDRAŽENOVIĆ, B., VESELIĆ, D., MITIĆ, Sampled Data Quasi-sliding Mode Control Strategies, IEEE International Conference on Industrial Technology, 2006, pp. 2640-2645.

26. BANDYOPADHYAY, B., D., FULWANI, High-performance Tracking Controller for Discrete Plant Using Nonlinear
Sliding Surface, IEEE Transactions on Industrial Electronics, vol. 56, no. 9, 2009, pp. 3628-3637.

27. MIJA, S., S., THOMAS, Reaching Law Based Sliding Mode Control For Discrete MIMO Systems, International Conference on Control, Automation, Robotics \& Vision, 2010, pp. 1291-1296.

28. NIU, Y., D. W. C., HO, Z., WANG, Improved Sliding Mode Control for Discrete-Time Systems via Reaching Law, IET Control Theory \& Applications, vol. 4, no. 11, 2010, pp. 2245-2251.

29. KURODE, S., B., BANDYOPADHYAY, P., GANDHI, Discrete Sliding Mode Control for a Class of Underactuated Systems, Annual Conference of the IEEE Industrial Electronics Society, 2011, pp. 3936-3941.

30. BARTOSZEWICZ, A., P. LEŚNIEWSKI, Reaching Law Based Sliding Mode Control for Communication Networks, IET Control Theory \& Applications, vol. 8, no. 17, 2014, pp. 1914-1920.

31. CHAKRABARTY, S., B., BANDYOPADHYAY, A Generalized Reaching Law for Discrete Time Sliding Mode Control, Automatica, vol. 52, no. 2, 2015, pp. 83-86.

32. JAIN, R., Congestion Control and Traffic Management in ATM Networks: Recent Advances and a Survey, Computer Networks and ISDN Systems, vol. 28, no. 13, 1996, pp. 1723-1738.

33. LEE, J. Y., B. G., KIM, E. B., LEE, Design of New ABR Congestion Control Algorithm Using Probabilistic Congestion Detection in ATM Networks, Electronics Letters, vol. 36, no. 2, 2000, pp. 178-179.

34. IMER, O, S., COMPAINS, T., BASAR, R., SRIKANT, Available Bit Rate Congestion Control in ATM Networks, IEEE Control Systems Magazine, vol. 21, no. 1, 2001, pp. 38-56.

35. LENGLIZ, I., F. KAMOUN, A Rate-based Flow Control Method for ABR Service in ATM Networks, Computer Net-works, vol. 34, no. 1, 2000, pp. 129-138.

36. BLANCHINI, F., R., LO CIGNO, R., TEMPO, Robust Rate Control for Integrated Services Packet Networks, 
IEEE/ACM Transactions on Networking, vol. 10, no. 5, 2002, pp. 644-652.

37. LABERTEAUX, K. P., C. E., ROHRS, P. J., ANTSAKLIS, A Practical Controller for Explicit Rate Congestion Control, IEEE Transactions on Automatic Control, vol. 47 , no. 6 , 2002, pp. 960-978.

38. SUN, D. H., Q. H., ZHANG, Z. C., MU, Single Parametric Fuzzy Adaptive PID Control and Robustness Analysis Based on the Queue Size of Network Node, International Conference on Machine Learning and Cybernetics, 2004, pp. 397-400.

39. MASCOLO, S., Congestion Control in High-Speed Communication Networks Using the Smith Principle, Automatica, vol. 35 , no. 12, 1999, pp. 1921-1935.

40. MASCOLO, S., Smith's Principle for Congestion Control in High-Speed Data Networks, IEEE Transactions on Automatic Control, vol. 45, no. 2, 2000, pp. 358-364.

41. GÓMEZ-STERN, F., J. M., FORNÉS, F., R., RUBIO, Dead-time Compensation for ABR Traffic Control over ATM networks, Control Engineering Practice, vol. 10, no. 5, 2002, pp. 481-491.

42. DE CICCO, L., S., MASCOLO, S. I., NICULESCU, Robust Stability Analysis of Smith Predictor-Based
Congestion Control Algorithms for Computer Networks, Automatica, vol. 47, no. 8,2011 , pp. 1685-1692.

43. BARTOSZEWICZ, A., J., ŻUK, Discrete-time Sliding Mode Flow Controller for Multi-Source SingleBottleneck Connection-Oriented Communication Networks, Journal of Vibration and Control, vol. 15, no. 11, 2009, pp. 1745-1760.

44. BABURAJ, P., B., BANDYOPADHYAY, Discrete-time Integral Sliding-mode Flow Control for Connection-oriented Communication Networks, International Conference on Control, Automation, Robotics \& Vision, 2012, pp. 205-210.

45. REN, T., Z., ZHU, H., YU, G. M., DIMIROVSKI, Integral Sliding Mode Controller Design for Congestion Problem in ATM Networks, International Journal of Control, vol. 86, no. 3, 2013, pp. 529-539.

46. BABURAJ, P., B., BANDYOPADHYAY, Finite-time Sliding Mode Flow Control Design via Reduced Model for a Connection-oriented Communication Network, Journal of The Franklin Institute, vol. 351 , no. 11,2014 , pp. 4960-4977. 
